\title{
The Shape of Space after WMAP data
}

\author{
Jean-Pierre Luminet \\ Laboratoire Univers et Théories, CNRS-UMR 8102, Observatoire de Paris, F-92195 Meudon cédex, France
}

Received on 5 January, 2005

\begin{abstract}
What is the shape of space is a long-standing question in cosmology. I review recent advances in cosmic topology since it has entered a new era of experimental tests. High redshift surveys of astronomical sources and accurate maps of the Cosmic Microwave Background radiation (CMB) are beginning to hint at the shape of the universe, or at least to limit the wide range of possibilities. Among those possibilites are surprising "wrap around" universe models in which space, whatever its curvature, may be smaller than the observable universe and generate topological lensing effects on a detectable cosmic scale. In particular, the recent analysis of CMB data provided by the WMAP satellite suggest a finite universe with the topology of the Poincare dodecahedral spherical space. Such a model of a "small universe", the volume of which would represent only about $80 \%$ the volume of the observable universe, offers an observational signature in the form of a predictable topological lens effect on one hand, and rises new issues on the early universe physics on the other hand.
\end{abstract}

Keywords: Cosmology; Models of universe

\section{THE SHAPE OF THE UNIVERSE}

The problem of the global shape of the universe can be decomposed into three intertwined questions.

First, what is the space curvature? In homogeneous isotropic models of relativistic cosmology, there are only three possible answers. Three-dimensional space sections of spacetime may have zero curvature on the average - in such a case, two parallel lines keep a constant space separation and never meet, as in usual Euclidean space, sometimes called "flat space". Or space sections can be negatively curved, such as two any parallels diverge and never meet (such a space is the three-dimensional analogue of the Lobachevsky hyperbolic plane). Eventually, they can be positively curved, in which case all parallels reconverge and cross again (like on the twodimensional surface of a sphere).

The property for physical space to correspond to one of these three possibilities depends on the way the total energy density of the Universe may counterbalance the kinetic energy of the expanding space. The normalized density parameter $\Omega_{0}$, defined as the ratio of the actual density to the critical value that an Euclidean space would require, characterizes the present-day contents (matter and all forms of energy) of the Universe. If $\Omega_{0}$ is greater than 1 , then space curvature is positive and geometry is spherical; if $\Omega_{0}$ is smaller than 1 the curvature is negative and geometry is hyperbolic; eventually $\Omega_{0}$ is strictly equal to 1 and space is Euclidean.

The second question about the shape of the Universe is to know whether space is finite or infinite - equivalent to know whether space contains a finite or an infinite amount of matter-energy, since the usual assumption of homogeneity implies a uniform distribution of matter and energy through space. From a purely geometrical point of view, all positively curved spaces (called spherical spaces whatever their topology) are finite, but the converse is not true : flat (Euclidean) or negatively curved (hyperbolic) spaces can have finite or infinite volumes, depending on their degree of connectedness (Ellis, 1971 ; Lachièze-Rey \& Luminet, 1995). For instance, in a flat space with cubic torus topology, as soon as a particle or a light ray "exits" a given face of the fundamental cube, it "re-enters" from the opposite face, so that space is finite, although without a boundary.

From an observable point of view, it is necessary to distinguish between the "observable universe", which is the interior of a sphere centered on the observer and whose radius is that of the cosmological horizon (roughly the radius of the last scattering surface), and the physical space. Again there are only three logical possiblities. First, the physical space is infinite - like for instance the simply-connected Euclidean space. In this case, the observable universe is an infinitesimal patch of the full universe and, although it has long been the preferred model of many cosmologists, this is not a testable hypothesis. Second, physical space is finite (e.g. an hypersphere or a closed multiconnected space), but greater than the observable space. In that case, one easily figures out that if physical space is much greater that the observable one, no signature of its finitude will show in the observable data. But if space is not too large, or if space is not globally homogeneous (as is permitted in many space models with multiconnected topology) and if the observer occupies a special position, some imprints of the space finitude could be observable. Third, physical space is smaller than the observable universe. Such an apparently odd possibility is due to the fact that space can be multiconnected and have a small volume. There a lot of geometrical possibilites, whatever the curvature of space. As it is well-known, such "small universe" models may generate multiple images of light sources, in such a way that the hypothesis can be tested by astronomical observations.

The third question about the shape of the Universe deals with its global topological properties (see Luminet, 2001 for a non-technical book about all the aspects of topology and its applications to cosmology). It is interesting to point out that none of these global properties is given by Einstein's field equations, since they are partial differential equations describing only the local, metric structure of spacetime (Friedmann, 1924). The present-day topology and curvature of space take likely their origin in the early quantum conditions of the Universe, which also governed its time evolution. The topologi- 
cal classification of homogeneous Riemannian 3-D spaces has made considerable progress during the last century. There are 18 Euclidean spaceforms (for a full description, see Riazuelo et al., 2004), a countable infinity of spherical spaceforms (see Gausmann et al, 2001) and a non-countable infinity of hyperbolic spaceforms (see Weeks, 1999.)

\section{COSMIC CRYSTALLOGRAPHY}

The topology and the curvature of space can be studied by using specific astronomical observations. For instance, from Einstein's field equations, the space curvature can be deduced from the experimental values of the total energy density and of the expansion rate. If the Universe was finite and small enough, we should be able to see "all around" it, because the photons might have crossed it once or more times. In such a case, any observer might identify multiple images of a same light source, although distributed in different directions of the sky and at various redshifts, or to detect specific statistical properties in the apparent distribution of faraway sources such as galaxy clusters. To do this, methods of "cosmic crystallography" have been devised (Lehoucq et al., 1996, 1999, 2000), and extensively studied by the Brazilian school of cosmic topology (Gomero et al., 2000, 2001a, 2002a,b, 2003; Fagundes \& Gausmann, 1999); see also Marecki et al. (2005).

Basically, cosmic crystallography looks at the 3dimensional apparent distribution of high redshift sources (e.g. galaxy clusters, quasars) in order to discover repeating patterns in the universal covering space, much like the repeating patterns of atoms observed in a crystal. "Pair Separation Histograms" (PSH) are in most cases able to detect a multiconnected topology of space, in the form of sharp spikes standing out above the noise distribution that is expected in the simply-connected case. Figures 1-3 visualize the "topological lens effect" generated by a multiconnected shape of space, and the way the topology can be determined by the PSH method.

However it was shown (Lehoucq et al., 2000; Gomero et al., 2002b ) that PSH may provide a topological signal only when the holonomy group of space has Clifford translations, a property which excludes all hyperbolic spaces.

\section{SPHERICAL LENSING}

In the first investigations of cosmic topology, the search for the shape of space had focused on big bang models with flat or negatively curved spatial sections. Since 1999 however, a combination of astronomical (type I supernovae) and cosmological (temperature anisotropies of the CMB) observations suggest that the expansion of the universe is accelerating, and constrain the value of space curvature in a range which marginally favors a positively curved (i.e. spherical) model. As a consequence, spherical spaceforms have come back to the forefront of cosmology.

Gausmann et al. (2001) have investigated the full properties of spherical universes. The simplest case is the celebrated hy-

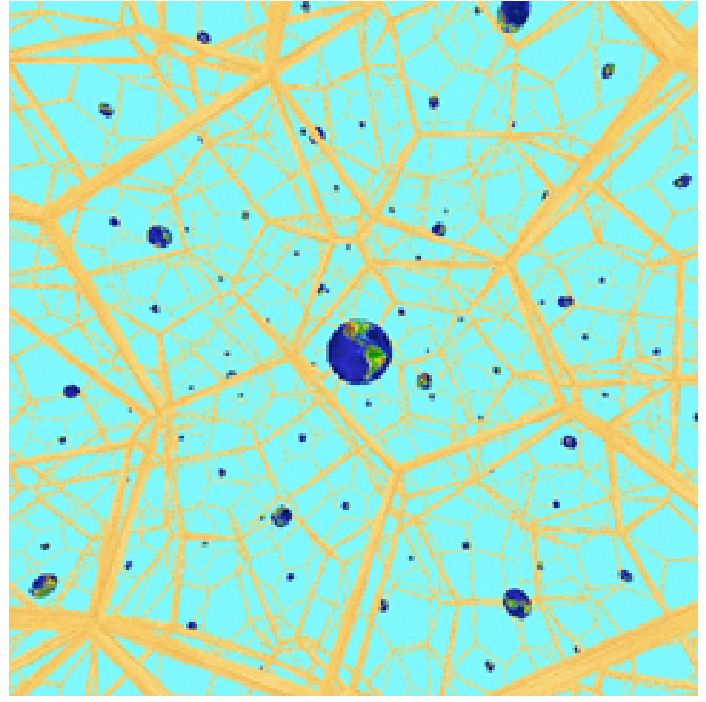

FIG. 1: In a multi-connected Universe, the physical space is identified to a fundamental polyhedron, the duplicate images of which form the observable universe. Representing the structure of apparent space is equivalent to representing its "crystalline" structure, each cell of which is a duplicate of the fundamental polyhedron. Here is depicted the closed hyperbolic Weeks space (only one celestial object is depicted, namely the Earth). As viewed from inside, it gives the illusion of a cellular space, tiled par polyhedra distorted with optical illusions (courtesy Jeffrey Weeks).

persphere, which is finite yet with no boundary. Actually there are an infinite number of spherical spaceforms, including lens spaces, prism spaces and polyhedral spaces. Gausmann et al. (2001) gave the construction and complete classification of such spaces, and discussed which topologies were likely to be detectable by crystallographic methods. They predicted the shapes of the pair separation histograms and they checked their predictions by computer simulations.

In addition, Weeks et al. (2003) and Gomero et al. (2001b) proved that the spherical topologies would be more easily detectable observationally than hyperbolic or flat ones. The reason is that, no matter how close space is to perfect flatness, only a finite number of spherical shapes are excluded by observational constraints. Due to the special structure of spherical spaces, topological imprints would be potentially detectable within the observable universe. Thus cosmologists are taking a renewed interest in spherical spaces as possible models for the physical universe.

\section{THE UNIVERSE AS A DRUMHEAD}

The main limitation of cosmic crystallography is that the presently available catalogs of observed sources at high redshift are not complete enough to perform convincing tests (Luminet and Roukema, 1998).

Fortunately, the topology of a small Universe may also be detected through its effects on such a "Rosetta stone" of cosmology as is the CMB fossil radiation (Levin, 2002 ; Riazuelo 


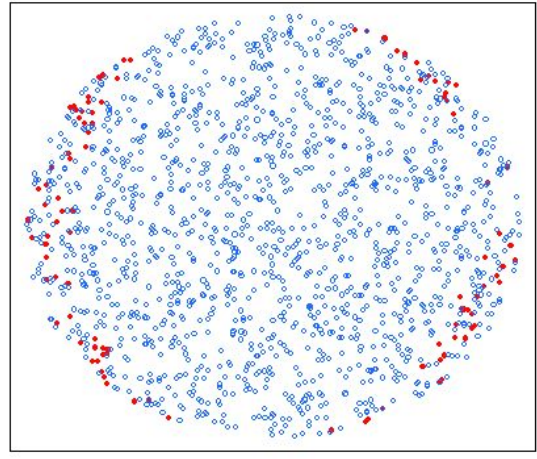

FIG. 2: Sky map simulation in hypertorus flat space (left). The fundamental polyhedron is a cube with length $=60 \%$ the horizon size and contains 100 "original" sources (dark dots). One observes 1939 topological images (light dots).

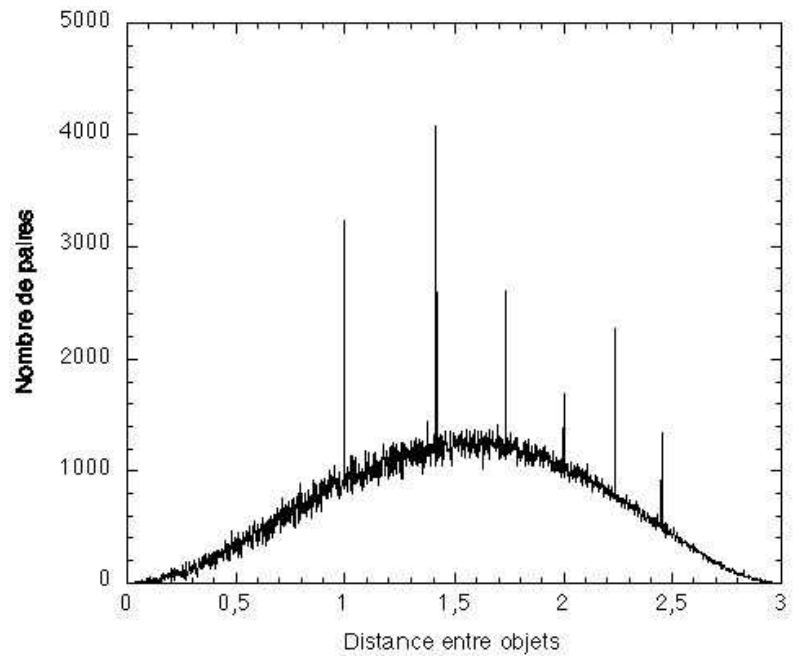

FIG. 3: The Pair Separation Histogram corresponding to Figure 2 exhibits spikes which stand out at values and with amplitudes depending on the topological properties of space.

et al., 2004a).

If you sprinkle fine sand uniformly over a drumhead and then make it vibrate, the grains of sand will collect in characteristic spots and figures, called Chladni patterns. These patterns reveal much information about the size and the shape of the drum and the elasticity of its membrane. In particular, the distribution of spots depends not only on the way the drum vibrated initially but also on the global shape of the drum, because the waves will be reflected differently according to whether the edge of the drumhead is a circle, an ellipse, a square, or some other shape.

In cosmology, the early Universe was crossed by real acoustic waves generated soon after the big bang. Such vibrations left their imprints 380000 years later as tiny density fluctuations in the primordial plasma. Hot and cold spots in the present-day $2.7 \mathrm{~K} \mathrm{CMB}$ radiation reveal those density fluctuations. Thus the CMB temperature fluctuations look like Chladni patterns resulting from a complicated threedimensional drumhead that vibrated for 380000 years. They yield a wealth of information about the physical conditions that prevailed in the early Universe, as well as present geometrical properties like space curvature and topology. More precisely, density fluctuations may be expressed as combinations of the vibrational modes of space, just as the vibration of a drumhead may be expressed as a combination of the drumhead's harmonics. The shape of space can be heard in a unique way. Lehoucq et al. (2002) calculated the harmonics (the socalled "eigenmodes of the Laplace operator") for most of the spherical topologies, and Riazuelo et al. (2004b) did the same for all 18 Euclidean spaces. Then, starting from a set of initial conditions fixing how the universe originally vibrated (the so-called Harrison-Zeldovich spectrum), they evolved the harmonics forward in time to simulate realistic CMB maps for a number of flat and spherical topologies (Uzan et al., 2003a).

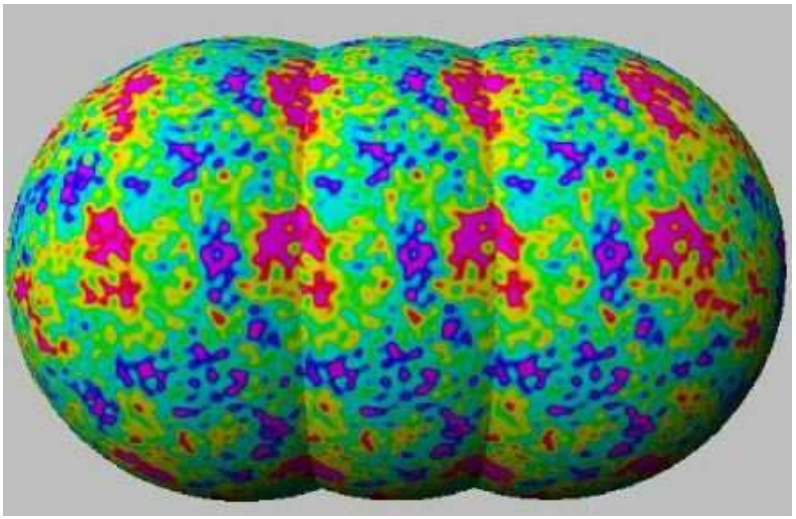

FIG. 4: A multiconnected topology translates into the fact that any object in space may possess several copies of itself in the observable Universe. For an extended object like the region of emission of the $\mathrm{CMB}$ radiation we observe (the so-called last scattering surface) it can happen that it intersects with itself along pairs of circles. In this case, this is equivalent to say that an observer (located at the center of the last scattering surface) will see the same region of the Universe from different directions. As a consequence, the temperature fluctuations will match along the intersection of the last scattering surface with itself, as illustrated in the above figure. This CMB map is simulated for a multiconnected flat space - namely a cubic hypertorus whose length is 3.17 times smaller than the diameter of the last scattering surface. Only two duplicates are depicted.

\section{Primordial fluctuations}

The "concordance model" of cosmology describes the Universe as a flat infinite space in eternal expansion, accelerated under the effect of a repulsive "dark energy". The data collected by the NASA satellite WMAP (Bennett et al, 2003 ; 


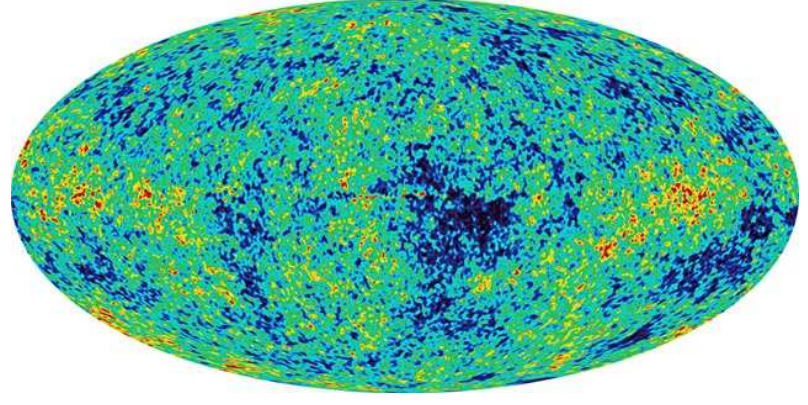

FIG. 5: Map of temperature anisotropies of CMB as observed by WMAP telescope. WMAP Homepage : http://map.gsfc.nasa.gov

Spergel et al., 2003) has recently produced a high resolution map of the CMB which showed the seeds of galaxies and galaxy clusters (Fig. 5) and allowed to check the validity of the dynamic part of the expansion model. However, combined with other astronomical data (Tonry et al., 2003), they suggest a value of the density parameter $\Omega_{0}=1.02 \pm 0.02$ at the $1 \sigma$ level. The result is marginally compatible with strictly flat space sections. Improved measurements could indeed lower the value of $\Omega_{0}$ closer to the critical value 1 , or even below to the hyperbolic case. Presently however, taken at their face value, WMAP data favor a positively curved space, necessarily of finite volume since all spherical spaceforms possess this property. This provides (provisory) answers to the first two questions stated above.

Now what about space topology ? There is an intriguing feature in WMAP data, already present in previous COBE mearurements (Hinshaw et al., 1996), although at a level of precision that was not significant enough to draw firm conclusions. The power spectrum of temperature anisotropies (Fig. 6) exhibits a set of "acoustic" peaks when anisotropy is measured on small and mean scales (i.e. concerning regions of the sky of relatively modest size). These peaks are remarkably consistent with the infinite flat space hypothesis. However, at large angular scale (for CMB spots typically separated by more than $60^{\circ}$ ), there is a strong loss of power which deviates significantly from the predictions of the concordance model. Thus it is necessary to look for an alternative.

CMB temperature anisotropies essentially result from density fluctuations of the primordial Universe : a photon coming from a denser region will loose a fraction of its energy to compete against gravity, and will reach us cooler. On the contrary, photons emitted from less dense regions will be received hotter. The density fluctuations result from the superposition of acoustic waves which propagated in the primordial plasma. Riazuelo et al. (2004a) have developed complex theoretical models to reproduce the amplitude of such fluctuations, which can be considered as vibrations of the Universe itself. In particular,they simulated high resolution CMB maps for various space topologies (Riazuelo et al., 2004b ; Uzan et al., 2003a) and were able to compare their results with real WMAP data. Depending on the underlying topology, the distribution of the fluctuations differs. For instance, in an infinite flat space, all wavelengths are allowed, and fluctuations must be present at

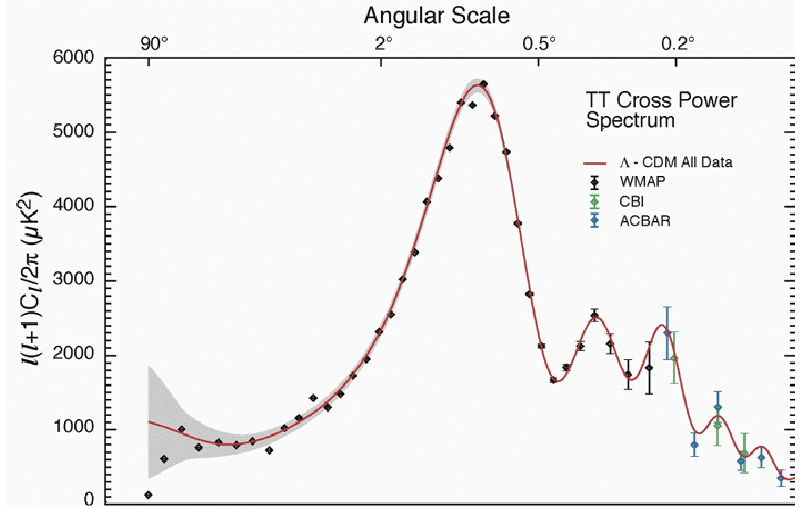

FIG. 6: The CMB power spectrum depicts the minute temperature differences on the last scattering surface, depending on the angle of view. It shows a series of peaks corresponding to small angular separations (the position and amplitude of the main peak allows us to measure space curvature), but at larger angular scales, peaks disappear. According to the predictions of the concordance model (continuous curve), at such scales the power spectrum should follow the so-called "Sachs-Wolfe plateau". However, WMAP measurements in this region (black diamonds) fall well below the plateau for the quadrupole and the octopole moments (first two diamonds on the left). While the flat infinite space model cannot explain this feature, multiconnected space models with a "well-proportioned" topology are remarkably consistent with such data. WMAP Homepage : http://map.gsfc.nasa.gov

all scales.

\section{COSMIC HARMONICS}

The CMB temperature fluctuations can be decomposed into a sum of spherical harmonics, much like the sound produced by a music instrument may be decomposed into ordinary harmonics. The "fundamental" fixes the height of the note (as for instance a 440 hertz acoustic frequency fixes the $A$ of the pitch), whereas the relative amplitudes of each harmonics determine the tone quality (such as the $A$ played by a piano differs from the $A$ played by a harpsichord). Concerning the relic radiation, the relative amplitudes of each spherical harmonics determine the power spectrum, which is a signature of the geometry of space and of the physical conditions which prevailed at the time of CMB emission.

The first observable harmonics is the quadrupole (whose wavenumer is $\ell=2$ ). WMAP has observed a value of the quadrupole 7 times weaker than expected in a flat infinite Universe. The probability that such a discrepancy occurs by chance has been estimated to $0.2 \%$ only. The octopole (whose wavenumber is $\ell=3$ ) is also weaker ( $72 \%$ of the expected value). For larger wavenumbers up to $\ell=900$ (which correspond to temperature fluctuations at small angular scales), observations are remarkably consistent with the standard cosmological model.

The unusually low quadrupole value means that long wave- 
lengths are missing. Some cosmologists have proposed to explain the anomaly by still unknown physical laws of the early universe (Tsujikawa et al., 2003). A more natural explanation may be because space is not big enough to sustain long wavelengths. Such a situation may be compared to a vibrating string fixed at its two extremities, for which the maximum wavelength of an oscillation is twice the string length. On the contrary, in an infinite flat space, all the wavelengths are allowed, and fluctuations must be present at all scales. Thus this geometrical explanation relies on a model of finite space whose size smaller than the observable universe constrains the observable wavelengths below a maximum value.

\section{WELL-PROPORTIONED SPACES}

Such a property has been known for a long time, and was used to constrain the topology from COBE observations (Sokolov, 1993 ; Starobinsky, 1993). Preliminary oversimplified analyses (Stevens et al., 1993 ; de Oliveira-Costa \& Smoot, 1995) suggested that any multi-connected topology in which space was finite in at least one space direction had the effect of lowering the power spectrum at large wavelengths. Weeks et al. (2004) reexamined the question and showed that indeed, some finite multiconnected topologies do lower the large-scale fluctuations whereas others may elevate them. In fact, the long wavelengths modes tend to be relatively lowered only in a special family of closed multiconnected spaces called "well-proportioned". Generally, among spaces whose characteristic lengths are comparable with the radius of the last scattering surface $R_{l s s}$ (a necessary condition for the topology to have an observable influence on the power spectrum), spaces with all dimensions of similar magnitude lower the quadrupole more heavily than the rest of the power spectrum. As soon as one of the characteristic lengths becomes significantly smaller or greater than the other two, the quadrupole is boosted in a way not compatible with WMAP data. The property was proved geometrically (Weeks et al., 2004), and checked out by numerical simulations (Riazuelo et al., 2004a). In the case of flat tori, they have varied their proportions and shown that a cubic torus lowers the quadrupole whereas an oblate or a prolate torus increase the quadrupole. They have also studied spherical spaces and shown that polyhedric spaces suppress the quadrupole whereas high order lens spaces (strongly anisotropic) boost the quadrupole. Thus, well-proportioned spaces match the WMAP data much better than the infinite flat space model.

\section{THE POINCARÉ DODECAHEDRAL SPACE}

Among the family of well-proportioned spaces, the best fit to the observed power spectrum is the Poincaré Dodecahedral Space (hereafter PDS) (Luminet et al., 2003).

PDS may be represented by a dodecahedron (a regular polyhedron with 12 pentagonal faces) whose opposite faces are glued after a $36^{\circ}$ twist (Fig. 7). Such a space is positively curved, and is a multiconnected variant of the simply- connected hypersphere $S^{3}$, with a volume 120 times smaller. A rocket going out of the dodecahedron by crossing a given face immediately re-enters by the opposite face. Propagation of light rays is such that any observer whose line-of-sight intercepts one face has the illusion to see inside a copy of his own dodecahedron (Fig. 8).

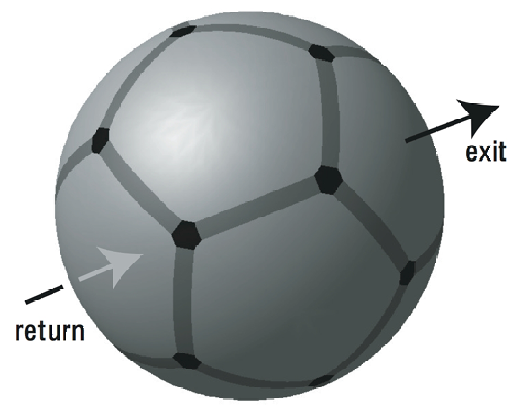

FIG. 7: Poincaré Dodecahedral Space can be described as the interior of a spherical dodecahedron such that when one goes out from a pentagonal face, one comes back immediately inside the space from the opposite face, after a $36^{\circ}$ rotation. Such a space is finite, although without edges or boundaries, so that one can indefinitely travel within it.

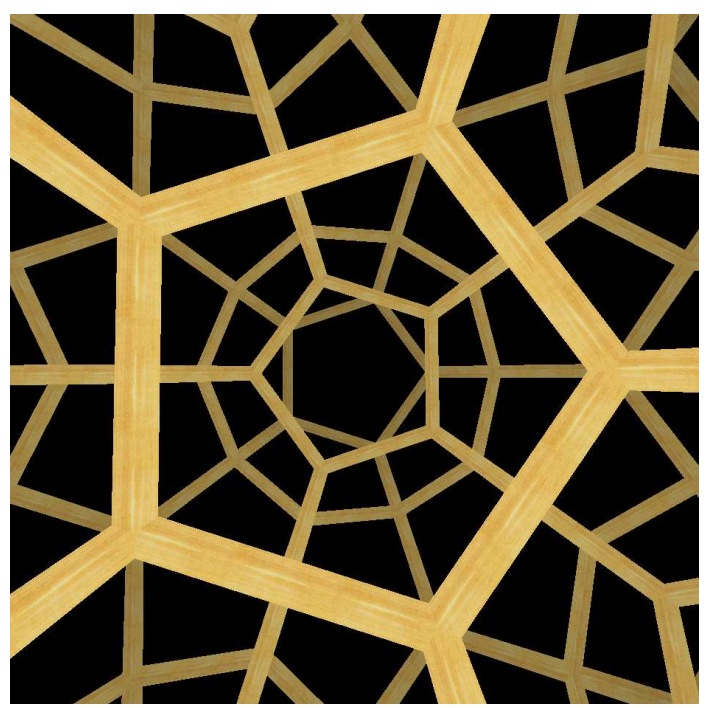

FIG. 8: View from inside PDS perpendicularly to one pentagonal face. In such a direction, ten dodecahedra tile together with a 1/10th turn to tessellate the universal covering space $S^{3}$. Since the dodecahedron has 12 faces, 120 dodecahedra are necessary to tessellate the full hypersphere. Thus, an observer has the illusion to live in a space 120 times vaster, made of tiled doecahedra which duplicate like in a mirror hall (courtesy Jeffrey Weeks).

The associated power spectrum, namely the repartition of fluctuations as a function of their wavelengths corresponding to PDS, strongly depends on the value of the mass-energy density parameter. Luminet et al. (2003) computed the CMB mul- 
tipoles por $\ell=2,3,4$ and fitted the overall normalization factor to match the WMAP data at $\ell=4$, and then examined their prediction for the quadrupole and the octopole as a function of $\Omega_{0}$. There is a small interval of values within which the spectral fit is excellent, and in agreement with the value of the total density parameter deduced from WMAP data $(1.02 \pm 0.02)$. The best fit is obtained for $\Omega_{0}=1.016$ (Fig. 9). The result is quite remarkable because the Poincaré space has no degree of freedom. By contrast, a 3-dimensional torus, constructed by gluing together the opposite faces of a cube and which constitutes a possible topology for a finite Euclidean space, may be deformed into any parallelepiped : therefore its geometrical construction depends on 6 degrees of freedom.

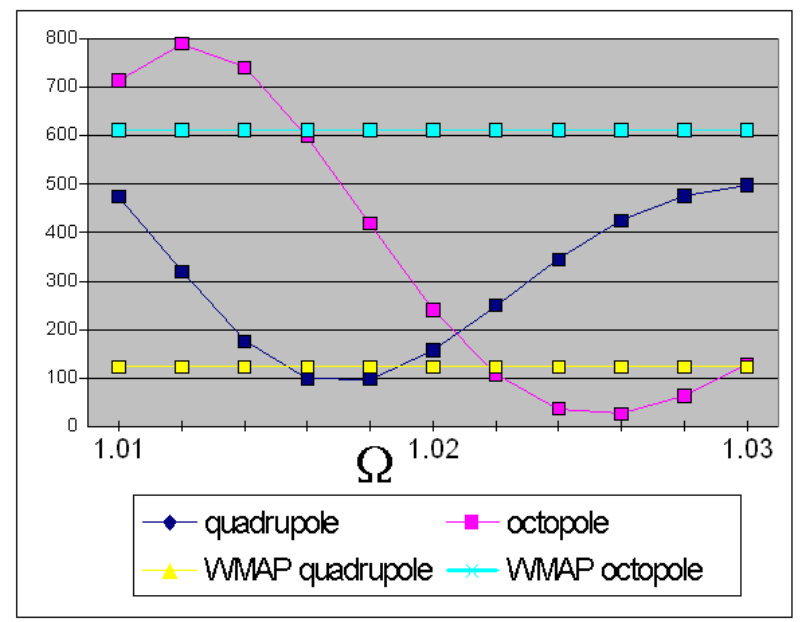

FIG. 9: The values of the total mass-energy density parameter (assuming $\Omega_{m}=0.28$ ) for which the Poincaré Dodecahedral Space fits the WMAP observations.

The values of the matter density $\Omega_{m}$, of the dark energy density $\Omega_{\lambda}$ and of the expansion rate $\mathrm{H}_{0}$ fix the radius of the last scattering surface $R_{l s s}$ as well as the curvature radius of space $R_{c}$, thus dictate the possibility to detect the topology or not. For $\Omega_{m}=0.28, \Omega_{0}=1.016$ and $\mathrm{H}_{0}=62 \mathrm{~km} / \mathrm{s} / \mathrm{Mpc}$, $R_{l s s} \sim 53 \mathrm{Gpc}$ and $R_{c}=2.63 R_{l s s}$. It is to be noticed that the curvature radius $R_{c}$ is the same for the simply-connected universal covering space $S^{3}$ and for the multiconnected PDS. Incidently, the numbers above show that, contrary to a current opinion, a cosmological model with $\Omega_{0} \sim 1.02$ is far from being "flat" (i.e. with $R_{c}=\infty$ )! For the same curvature radius, PDS has a volume 120 times smaller than $S^{3}$. Therefore, the smallest dimension of the fundamental dodecahedron is only $43 \mathrm{Gpc}$, and its volume about $80 \%$ the volume of the observable universe (namely the volume of the last scattering surface). This implies that some points of the last scattering surface will have several copies. Such a lens effect is purely attributable to topology and can be precisely calculated in the framework of the PDS model. It provides a definite signature of PDS topology, whereas the shape of the power spectrum gives only a hint for a small, well-proportioned universe model.

To resume, the Poincaré Dodecahedral Space accounts for the low value of the quadrupole as observed by WMAP in the fluctuation spectrum, and provides a good value of the octopole. To be confirmed, the PDS model, which has been popularized as the "soccerball universe model", must satisfy two experimental tests :

1) A finer analysis of WMAP data, or new data from the future European satellite "Planck Surveyor" (scheduled 2007), will be able to determine the value of the energy density parameter with a precision of $1 \%$. A value lower than 1.01 will discard the Poincare space as a model for cosmic space, in the sense that the size of the corresponding dodecahedron would become greater than the observable universe and would not leave any observable imprint on the $\mathrm{CMB}$, whereas a value greater than 1.01 would strengthen its cosmological pertinence.

2) If space has a non trivial topology, there must be particular correlations in the CMB, namely pairs of "matched circles" along which temperature fluctuations should be the same (Cornish et al, 1998). The PDS model predicts 6 pairs of antipodal circles with an angular radius less than $35^{\circ}$.

Such circles have been searched in WMAP data by two different teams, using various statistical indicators and massive computer calculations. On the one hand, Cornish et al. (2004) claimed to have found no matched circles on angular sizes greater than $25^{\circ}$, and thus rejected the PDS hypothesis. Moreover, they claimed that any reasonable topology smaller than the horizon was excluded. This is a wrong statement because they searched only for antipodal or nearly-antipodal matched circles. However Riazuelo et al. (2004b) have shown that for generic topologies (including the well-proportioned topologies which are good candidates for explaining the WMAP power spectrum), the matched circles are not back-to-back and space is not globally homogeneous, so that the positions of the matched circles depend on the observer's position in the fundamental polyhedron. The corresponding larger number of degrees of freedom for the circles search in the WMAP data generates a dramatic increase of the computer time, up to values which are out-of-reach of the present facilities.

On the other hand, Roukema et al. (2004) performed the same analysis for smaller circles, and found six pairs of matched circles distributed in a dodecahedral pattern, each circle on an angular size about $11^{\circ}$. This implies $\Omega_{0}=$ $1.010 \pm 0.001$ for $\Omega_{m}=0.28 \pm 0.02$, values which are perfectly consistent with the PDS model.

It follows that the debate about the pertinence of PDS as the best fit to reproduce CMB observations is fully open. Since then, the properties of PDS have been investigated in more details by various authors. Lachièze-Rey (2004) found an analytical expression of the eigenmodes of PDS, whereas Aurich et al. (2005) computed numerically the first 10521 eigenfunctions up to the $\ell=155$ mode and also supported the PDS hypothesis for explaining WMAP data. Eventually, the secondyear WMAP data, originally expected by February 2004 but delayed for at least one year due to unexpected surprises in the results, may soon bring additional support to a spherical multiconnected space model. 


\section{CONSEQUENCES FOR THE PHYSICS OF THE EARLY UNIVERSE}

Finite well-proportioned spaces, and specially the Poincaré dodecahedral spherical space, open something like a "Pandora box" for the physics that prevailed in the early universe. The concordance model relies mostly on the hypothesis that the early universe underwent a phase of exponential expansion the celebrated "inflationary process". Even without mentioning topological subtleties, it is good to recall that inflation theory gets into some troubles. In the simplest inflationary models, space is supposed to have become immensely larger than the observable universe after its phase of exponential growth. Therefore apositive curvature (i.e. $\Omega_{0}>1$ ), even weak, implies a finite space and sets strong constraints on the number of e-foldings that took place during an inflation phase. It is possible to build models of "low scale" inflation where the inflationary phase is short and leads to a detectable space curvature (Uzan et al., 2003b). It turns out that, if space is not flat, the possibility of a multiconnected topology is not in contradiction with the general idea of inflation, due a number of free and adjustable parameters in this kind of models. Yet, no convincing physical scenario has been proposed (see however Linde, 2003).

In most cosmological models, it is generally assumed that spatial homogeneity stays valid beyond the horizon scale. For instance, in the model of chaotic inflation (Linde et al., 1994 ; Guth, 2000), the universe could be very homogeneous but on scales much larger than the horizon scale. On this respect, the PDS model seems incompatible with chaotic inflation : it requires only one expanding bubble universe, of size sufficlently small to be entirely observable. In his seminal cosmological paper, Einstein (1917) had already emphasized that spatially closed universes had the advantage to eliminate boundary conditions (Wheeler, 1968). A small universe like the PDS or a well-proportioned one, in which the observer could have access to all the existing physical reality is still more advantageous (Ellis \& Schreiber, 1986). It is the only type of model in which the astronomical future could be definitely predicted - such as the return of Halley's comet -, because only in such universes the observer could access to all the data in order to perform such predictions.

Maybe the most fundamental issue is to link the presentday topology of space to a quantum origin, since classical general relativity does not allow for topological changes during the course of cosmic evolution. Theories of quantum gravity could allow to address the problem of a quantum origin of space topology. For instance, in the approach of quantum cosmology, some simplified solutions of Wheeler-de Witt equations show that the sum over all topologies involved in the calculation of the wavefunction of the universe is dominated by spaces with small volumes and multiconnected topologies (Carlip, 1993 ; e Costa and Fagundes, 2001). In the approach of brane worlds (see Brax 2003 for a review), the extradimensions are often assumed to form a compact Calabi-Yau manifold ; in such a case, it would be strange that only the ordinary dimensions of our 3-brane would not be compact like the extra ones.
These are only heuristic indications on the way unified theories of gravity and quantum mechanics could "favor" multiconnected spaces. Whatsoever the fact that some particular multiconnected space models, such as PDS, may be refuted by future astronomical data, the question of cosmic topology will stay as a major question about the ultimate structure of our universe.

\section{References}

Aurich W., Lustig S., and Steiner F. (2005), astro-ph/0412569. Bennett, C. L. et al. (2003), Astrophys. J. Suppl. Ser. 148, $1-27$.

Brax P. and Van de Bruck C. (2003) Class. Quant. Grav. 20, R201-R232.

Carlip S. (1993), Class. Quant. Grav. 10, 207-218.

Cornish, N., Spergel, D. and Starkman, G. (1998), Class. Quant. Grav. 15, 2657-2670.

Cornish, N. J., Spergel, D. N., Starkman, G. D. and Komatsu, E. (2004), Phys. Rev. Lett. 92, 201302.

de Oliveira-Costa A. and Smoot G.F. (1995), Astrophys. J. 448, 447.

e Costa, S. S. and Fagundes, H. V. (2001), Gen. Rel. Grav. 33, 1489-1494

Einstein, A. (1917), Preuss. Akad.Wiss. Berlin Sitzber. 142152.

Ellis, G. F. R. (1971), Gen. Rel. Grav. 2, 7-21.

Ellis, G. F. R. and Schreiber, W. (1986), Phys. Lett. A 115, 97-107.

Fagundes, H. V. and Gausmann E. (1999), Phys. Lett. A261, 235-239.

Friedmann, A. (1924), Z. Phys. 21, 326-332.

Gausmann E., Lehoucq R., Luminet J.-P., Uzan J.-P. and Weeks J. (2001), Class. Quant. Grav., 18, 5155.

Gausmann, E., Lehoucq, R., Luminet, J.-P., Uzan, J.-P. and Weeks, J. (2001), Classical and Quantum Gravity, 18, 1-32.

Gomero, G.I. (2003), Class. Quant. Grav. 20, 4775-4784.

Gomero, G.I., Reboucas, M.J., and Teixeira, A.F.F. (2000), Phys. Lett. A275, 355-367 ; (2001a), Class. Quant. Grav. 18, 1885-1906.

Gomero, G.I., Reboucas, M.J., Tavakol, R. (2001b), Class. Quant. Grav. 18, 4461-4476 ; (2002), Int. J. Mod. Phys. A17, 4261-4272.

Gomero, G.I., Teixeira, A.F.F., Reboucas, M.J., Bernui, A. (2002), Int. J. Mod. Phys. D11, 869-892.

Guth, A. H. (2000), Phys. Rep. 333, 555-574.

Hinshaw G. et al. (1996), Astrophys. J. Lett. 464, L17-20.

Lachièze-Rey, M. (2004), Class.Quant.Grav. 21, 2455-2464.

Lachièze-Rey, M. and Luminet, J. P. (1995), Phys. Rep. 254, 135-214.

Lehoucq R., Lachièze-Rey M. and Luminet J.P. (1996), Astron. Astrophys. 313, 339-346.

Lehoucq R., Luminet J.-P. and Uzan J.-P. (1999), Astron. Astrophys. 344, 735.

Lehoucq R., Uzan J.-P. and Luminet J.-P. (2000), Astron. Astrophys. 363, 1 . 
Lehoucq, R., Uzan, J.-P. and Weeks, J. (2003), Class. Quant. Grav. 20, 1529-1542.

Lehoucq, R., Weeks, J., Uzan, J.-P., Gausmann, E. and Luminet, J.-P. (2002), Classical and Quantum Gravity, 19, 46834708.

Levin J. (2002) Phys. Rep. 365, 251-333.

Linde A. (2003) JCAP 0305, 002.

Linde, A., Linde, D. and Mezhlumian, A. (1994), Phys. Rev. D 49, 1783-1826.

Luminet, J.- P., L'Univers chiffonné, Fayard, Paris, 2001. Portugese translation Sentido e segredos do Universo, Instituto Piaget, Lisboa, 2002.

Luminet J.-P. and Roukema B. (1998), Proc. Cargese 98 summer school Cosmology: The Universe at Large Scale, M. Lachieze-Rey (Ed.), Kluwer Ac. Pub., NATO ASI 970491.

Luminet J.-P., Weeks J., Riazuelo A., Lehoucq R. and Uzan J.-P. (2003), Nature 425, 593-595.

Marecki A., Roukema B., Bajtlik S. (2005), to appear in Astron. and Astrophys., astro-ph/0412181.

Riazuelo, A., Uzan, J.-P., Lehoucq, R. and Weeks, J. (2004a), Phys. Rev. D69, 103514.

Riazuelo, A., Weeks, J., Uzan, J.-P., Lehoucq, R. and Luminet, J.-P. (2004b), Phys. Rev. D69, 103518.
Roukema, B. F., Lew, B., Cechowska, M., Marecki, A. and Bajtlik, S. (2004), Astron. and Astrophy. 423, 821.

Sokolov I.Y. (1993), JETP Lett. 57, 617.

Spergel, D. N. et al. (2003), Astrophys. J. Suppl. Ser. 148, 175-194.

Starobinsky A.A.(1993), JETP Lett. 57, 622.

Stevens D., Scott D. and Silk J. (1993), Phys. Rev. Lett. 71 20.

Tonry et al., (2003) Astrophys.J. 594 1-24.

Tsujikawa, S.,Maartens, R. and Brandenberger, R. H. (2003), e-print astro-ph/0308169.

Uzan, J.-P., Riazuelo, A., Lehoucq, R. and Weeks, J. (2003a), astro-ph/0303580, to appear in Phys. Rev. D.

Uzan J.-P., Kirchner U., Ellis G.F.R. (2003b), Mon. Not. Roy. Astron. Soc. 344 L65.

Weeks, J., SnapPea: A computer program for creating and studying hyperbolic 3-manifolds, available by anonymous ftp from http://geometrygames.org/SnapPea/

Weeks, J., Lehoucq, R. and Uzan, J.-P. (2003), Class. Quant. Grav. 20, 1529-1542.

Weeks, J., Luminet, J.-P., Riazuelo, A. and Lehoucq, R. (2004), MNRAS, 352, 258-262.

Wheeler, J. A. Einstein's Vision (Springer, Berlin, 1968). 